\section{DISEASE MODELS}

\section{Brain}

\section{teasers}

\section{Cerebral organoids are helping researchers to develop fresh approaches to treating brain cancer.}

\section{BY ANNA NOWOGRODZKI}

$\mathrm{E}$ ven in comparison to other types of cancer, brain cancer is particularly deadly. People with glioblastoma multiforme, one of the most common forms of brain cancer, have a median survival of less than 15 months after diagnosis. The US Food and Drug Administration (FDA) has so far approved only five drugs for treating brain cancer. Given this limited range, researchers could search for potential treatments among the wider pool of all FDA-approved drugs; however, any found to be effective would probably work in just a slim percentage of people with brain cancer.

Unfortunately, those with the condition do not have time to cycle through hundreds of drugs to find the one that might work. But if researchers could grow numerous small brain-like structures that contained a replica of the person's tumour and then bathe them in various treatments, in the space of a few weeks, they might learn exactly which ones would have the best chance of fighting brain cancer in that individual. That's the vision of Howard Fine, a neuro-oncologist at Weill Cornell Medicine in New York City who is developing such models, known as cerebral organoids, for the study of brain cancer - with the ultimate goal of finding the most appropriate treatment for each person.

Organoids are miniature, laboratory-grown versions of the body's organs. They contain several cell types and have a simplified $3 \mathrm{D}$ anatomy. They are particularly valuable for studying brain cancer because neither human brain tumours transplanted into mice nor human tumour stem cells grown in a culture dish behave in the same way as their counterparts in the body.

At only five years old, the field of cerebral organoids is still young. Many challenges lie ahead, including how to give these organoids blood vessels, immune cells and a more realistic structure. But Fine and other researchers think that cerebral organoids might provide fresh opportunities for studying how tumours arise, screening drug candidates and developing evidence-based,

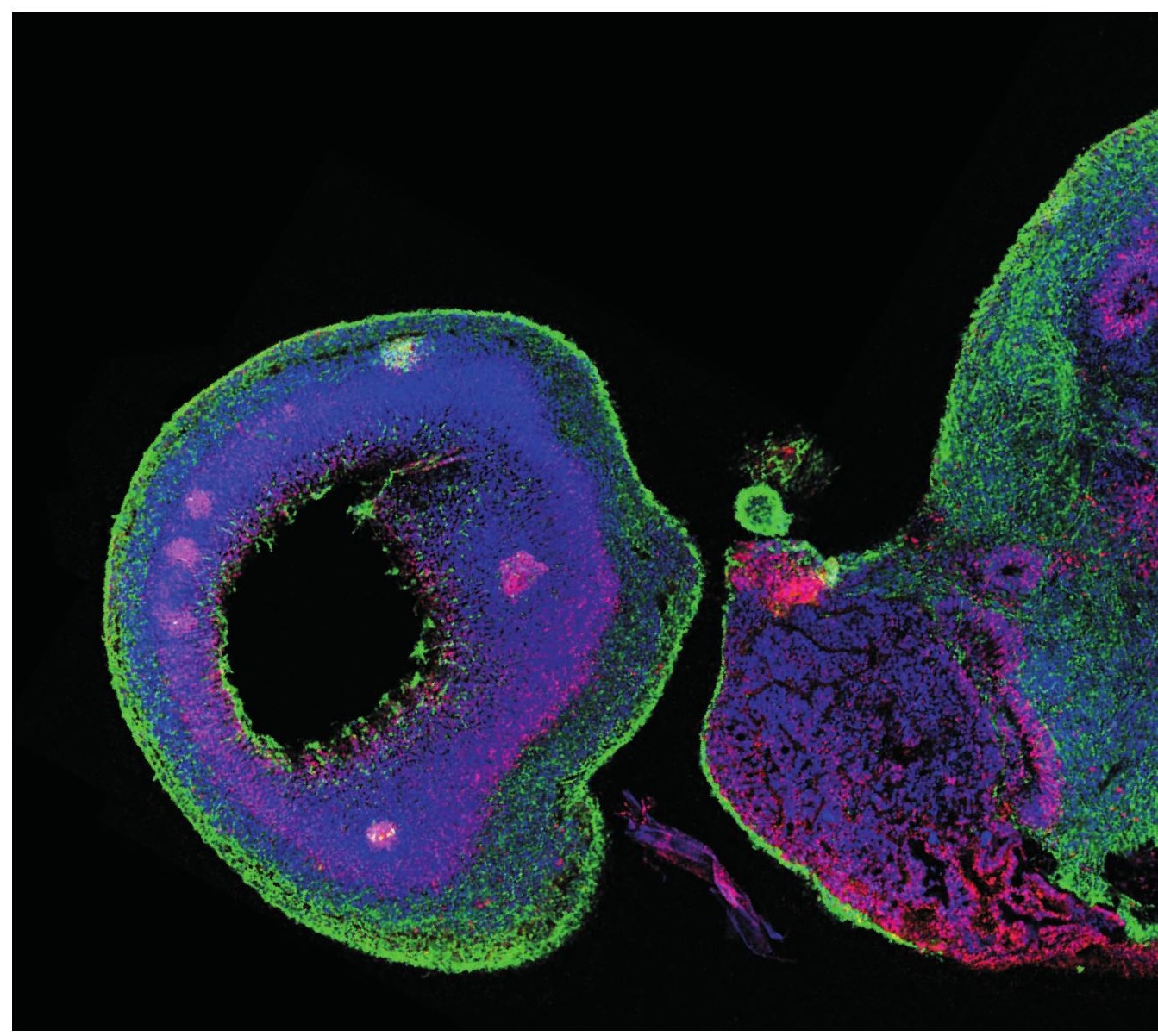

A section through a cerebral organoid reveals organized neurons (green) and neural stem cells (red).

personalized treatment plans for people with brain cancer.

\section{'AHA!' MOMENT}

The discovery of cerebral organoids was a happy accident. In 2011, Madeline Lancaster, a cell biologist then based at the Institute of Molecular Biotechnology of the Austrian Academy of Sciences in Vienna, was trying to grow 2D neural rosettes - circular arrangements of neural cells that are derived from embryonic stem cells.

But Lancaster, now at the MRC Laboratory of Molecular Biology in Cambridge, UK, had little experience of tissue culture. In some of the dishes, the cells did

\section{"We can introduce mutations almost identical to those seen in human patients."} not behave as she expected. They failed to stick to the plastic surface, and so rather than growing as flat rosettes, they rolled up into $3 \mathrm{D}$ floating balls with a self-organized structure. Seeing their potential, she began to deliberately grow and then develop these structures until they vaguely resembled human brain tissue. In 2013, she described the mini-brains (M. A. Lancaster et al. Nature 501, 373-379; 2013), which she had refined to include structures such as a cerebral cortex - and in so doing, launched the now burgeoning body of research on cerebral organoids.

Years earlier, Fine's team ran across a problem during their studies of brain cancer. The standard approach to modelling the condition in the lab had been to grow human tumour cells in a dish and then to transplant them into mouse brains. But for years, researchers had noticed that the resulting growths did not behave as they would in human brains. "The mouse brain is a very different microenvironment from the human brain," says Fine. The brain-tumour cells grown in Petri dishes stubbornly refused to form tumour stem cells, even after they were transplanted into mouse brains. Such stem cells are crucial for instigating both resistance to treatment and disease recurrence in humans.

So, in 2006, Fine's team developed what has become the standard way to grow tumour stem cells in the lab, a system they saw as a more realistic approach to modelling brain cancer in the lab (J. Lee et al. Cancer Cell 9, 391-403; 2006). But Fine, who is also a clinician, was able to observe first-hand how poorly even these improved models were serving his patients. At the start of his career in the 1980s, the median survival time was 12 months. Now, it is 15 months: a gain of only three months from three decades of research and drug development. Almost all of the 15,000-20,000 people who he has cared for with malignant glioma have died from the disease. "After 30 years," he 


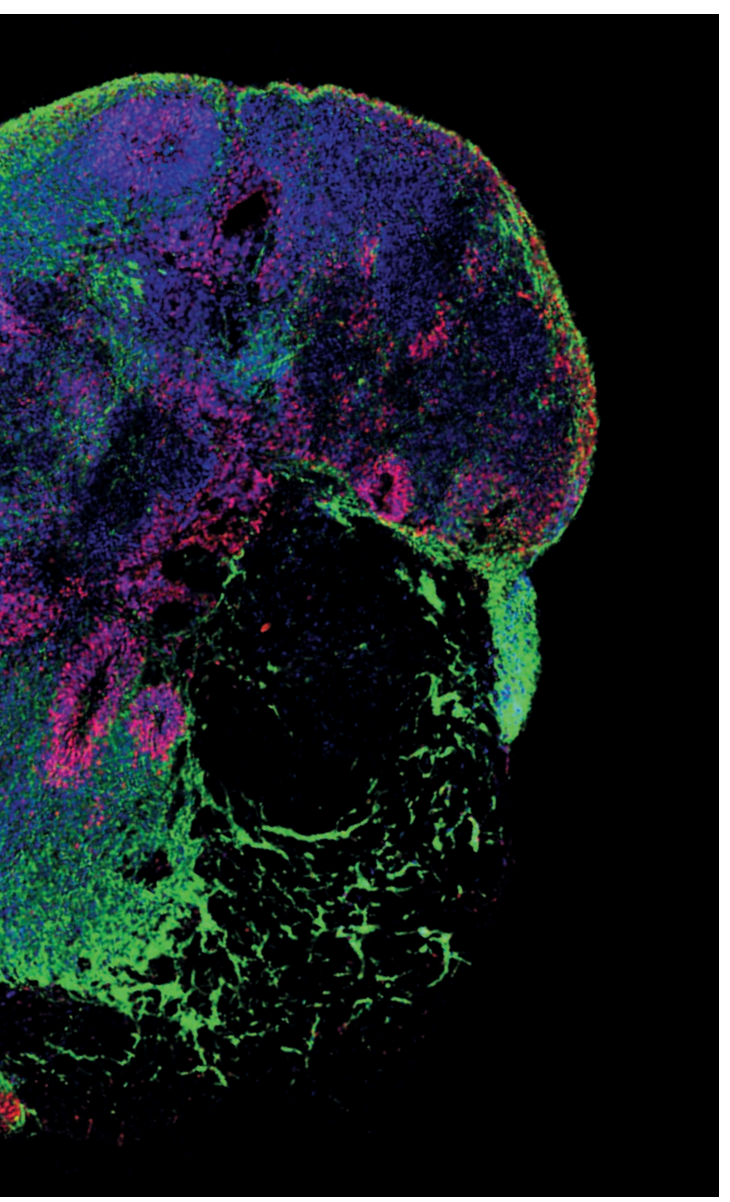

says, "it doesn't take a genius to figure out that what we're doing isn't working." He focused on what he saw as a considerable weakness in the development of therapies - that "model systems look nothing like the human disease that I was seeing in the clinic".

In the body, tumours are not isolated from other cells. They are part of organs that provide them with a scaffold on which to grow, as well as blood vessels through which they are supplied with oxygen, and tumour cells interact with the cells that surround them. But Fine's models lacked all such features. "The cells may be the right cells, but they're growing on plastic," he says.

Then Fine read Lancaster's paper on cerebral organoids. "It was an 'Aha!' moment," he says. "That's what we've been looking for."

\section{MINI-BRAINS IN THE MAKING}

Fine and others are now relying on these biological model systems. But although cerebral organoids are made up of brain cells and have a $3 \mathrm{D}$ structure, they are not yet miniature replicas of human brains. Researchers do not know how to ensure that important brain structures are formed in anatomically correct places, for instance. "Our cerebral organoids have almost all the major functional and neuroanatomical structures you'd find in a developing fetal brain, but their positioning is kind of random," Fine says. "If you were developing a whole body, your ear would be where your knee is." Cerebral organoids lack mechanisms that tell brain structures how to orient themselves when they begin to develop.

Imbuing these organoids with blood vessels and immune cells such as microglia is also a challenge. The absence of blood vessels means that cerebral organoids cannot be used to test treatments that target angiogenesis. Christopher Chen, a biomedical engineer at Boston University, Massachusetts, and at the Wyss Institute for Biologically Inspired Engineering at Harvard University in Boston, is collaborating with Fine's team to engineer cerebral organoids that incorporate blood vessels. "The brain is highly vascularized," he says. "That vasculature is really important for the growth of these cancers, their ability to escape and metastasize, and for people to be able to deliver therapeutics."

But cultivating this network of blood vessels is proving to be extremely difficult. It must be dense enough to reach each cell in the organoid; however, because cell connectivity is important for brain function, the cells in cerebral organoids are closely packed, making it trickier to supply them with blood than it is to supply those in other kinds of organoid. Another issue is that the direction of blood flow and the direction in which neurons connect are coordinated in the brain. "We don't know if blood vessels going in the wrong directions would confuse the ability of neurons to connect," says Chen. Researchers are also uncertain about how to get endothelial cells in blood vessels to form the blood-brain barrier (see page S46). So there is no obvious road map for adding blood vessels. "Right now, we're in the 'everything but the kitchen sink' phase," Chen says.

But even with these limitations, cerebral organoids still have value as a model system. For instance, they enable researchers to image tumour development in real time. Visualizing tumours in mice, by contrast, requires the animals to be killed, which stops tumours from developing further - and means that it is impossible to image the same tumours at various stages of development. Also, tumours that are inserted into cerebral organoids will destroy the organoids in 1-2 weeks, says Fine, as opposed to the 3-8 months it takes for such tumours to grow big enough to kill a mouse. Researchers who want to observe tumour development in organoids over a longer period can treat the growths with chemotherapy agents.

Some researchers have found that cerebral organoids are a perfect system in which to study the earliest stages of tumour development. Junko Ogawa, a molecular biologist at the Salk Institute for Biological Studies in La Jolla, California, used the gene-editing technology CRISPR-Cas9 to both introduce an oncogene and disrupt a tumour-suppressor gene in such organoids, causing tumours to form. Unlike Fine, Ogawa induces tumours to form within the organoids, rather than transplanting tumours into them. "We can introduce mutations almost identical to those seen in human patients," she says. Ogawa is now working to introduce tumours from other organs, such as the lungs, into cerebral organoids to study cancers that spread to the brain.

\section{ORGANOID DONOR}

Since 2016, Fine's team has been using standard human embryonic stem-cell lines to produce generic cerebral organoids, in which the researchers then grow tumours from specific patients. But about six months ago, the team received approval from Weill Cornell's institutional review board to make cerebral organoids from a person's own stem cells, and it has already begun to do so for some patients.

Patient-specific organoids open up the possibility of incorporating a patient's immune cells into these models, making them more realistic and also facilitating the study of immunotherapies (S42). Immune cells cannot be added to generic organoids because they recognize the organoid as being foreign. But personalized organoids enable researchers to explore a more fundamental question of cancer biology: whether a person's tumour would grow differently inside a generic host. It could be that there is something about the person with brain cancer that makes them particularly hospitable to their tumour.

Fine will soon have a chance to get to the bottom of this and similar mysteries. In 2016, he received a US\$7-million grant from the Starr Foundation, a private philanthropic foundation based in New York City, to build a high-throughput facility that will use organoids derived from patients, and that contain the patient's tumour, to screen drug candidates. He will then use the results to treat the same patients in the clinic.

The facility is set to open within the next two months for drug screening but not clinical work. Fine estimates that it will take his team a year to help the New York state government to develop regulations for such patient-specific organoid facilities and to obtain clinicallaboratory certification. By late 2019, he hopes to commence the first clinical trial involving patient-specific cerebral organoids. Its goal will be to identify which of the roughly 1,500 FDA-approved drugs or drug combinations is most effective at fighting each person's tumour.

The idea that brain tumours grown in cerebral organoids represent better models of human brain cancer is so far untested, as is the suggestion that patient-specific organoids will be more informative than generic ones. But it is a safe bet that even the process of investigating these hypotheses will help to answer some interesting questions - and also produce further ones - about how brain cancer interacts with its surroundings, why it thrives when it does, and what strategies might lead to its defeat.

Anna Nowogrodzki is a science writer based in Boston, Massachusetts. 